\title{
Tribunal del Jurado: Efectos de la Participación en el Cambio de Opinión y Conocimiento de sus Miembros
}

\section{Court of the Jury: Effects of the Participation in the Change of Opinion and Knowledge of its Members}

\author{
Miguel Ángel Soria, Rita Berger, Montserrat Yepes, Inés Lovelle y Virginia García \\ Universidad de Barcelona, España
}

\begin{abstract}
Resumen. El siguiente estudio se propone evaluar el cambio de opinión y conocimiento en los miembros de un jurado simulado ("jurado en la sombra") compuesto por 19 participantes voluntarios. Mediante un cuestionario creado "ad hoc" y administrado pre-post experiencia evaluamos las características individuales y de procedimiento. Los resultados revelaron una diferencia significativa en una mejor valoración de la defensa frente a la acusación, concederle más importancia al origen del delito y una mejora del conocimiento sobre el funcionamiento del jurado y sus funciones. Dichos resultados si bien muestran limitaciones muéstrales nos permiten establecer que existe un mejor conocimiento del jurado tras la experiencia vivida, pero no se producen modificaciones significativas en las opiniones.

Palabras clave: tribunal del jurado, veredicto judicial , opinión jurado, jurado simulado, psicología sentencias.
\end{abstract}

\begin{abstract}
The present study aims to assess the changes in, opinion and knowledge in the members of a shadow jury composed of 19 volunteer participants. We administered a questionnaire created "ad hoc" before and after the experience, to measure participants" and procedural characteristics. Results showed significant differences in a more positive evaluation of the defense versus the prosecution, give more consideration to the cause and origin of the crime, and an increase in knowledge about the jury and its functions. Although such results showed limitations due to the sample characteristics, they allowed us to establish that participants were more knowledgeable regarding the jury after the study, although there were no significant changes of opinion.

Keywords: jury duty, jury decision-making, juror values, mock jury, sentences psychology.
\end{abstract}

\section{Introducción}

El tribunal del jurado tiene un rol integral en la administración de justicia ya que de él depende el porvenir del acusado (e.g. Daftary-Kapur, Dumas y Penrod, 2010). Devine, Clayton, Dunford, Seying y Price (2001) realizaron un metaanálisis sobre los

La correspondencia sobre este artículo puede dirigirse al primer autor a Departamento de Psicología Social de la Universidad de Barcelona. Passeig Vall d'Hebron, 171. Edifici Ponent, 4t Planta. 0035 Barcelona. Email: msoria@ub.edu estudios en relación al jurado popular (petit jury). Según los autores, este tipo de estudios pueden agruparse en cuatro categorías de características: procedimiento, participantes, caso, y deliberación. Las categorías de procedimiento son aquellos factores o parámetros institucionales relacionados con el funcionamiento del jurado (tamaño, instrucciones, etcétera). Las características de los participantes corresponden a aquellas diferencias individuales relacionadas con los miembros del jurado, acusado, víctima, demandante, jueces, abogados, variables demo- 
gráficas, rasgos de personalidad y experiencia entre otros). Las características del caso hacen referencia a aquellas variables asociadas al juicio específico y caso enjuiciado (tipo de caso, evidencia, cargos presentados, etc.).

Por último, las características de la deliberación incluyen todas las facetas de la interacción de los jurados (distribución inicial de las preferencias del jurado, influencia interpersonal y participación, etc.).

A nivel de procedimiento, las instrucciones judiciales han sido las más estudiadas (e.g. Fitzgerald, 2000; ForsterLee, Horowitz y Bourgeois, 1993; Schuller y Rzepa, 2002) seguidas por la orientación pre-jurado (Phaw, Bradshaw y Thomas, 2005), y los procedifentos judiciales (Collet y Kovera, 2003). No obstante, la mayoría de investigaciones sobre el jurado se han centrado principalmente en el estudio de las variables relativas a los participantes y el proceso de deliberación, en específico la toma de decisión y aquellos factores que pueden influir en dicho proceso y por tanto en el veredicto.

Las investigaciones sobre las características de los participantes han estudiado variables como el sexo (e.g. Golding, Bradshaw, Dunlap y Hodell, 2007; Najdowski, 2010; Shaw y Skolnick, 2005;), la edad (e.g. Fitzgerald, 2000; Higgins, Heath, Grannemann, 2007; Newcombre y Bransgrove, 2007), la raza (e.g. Abshire y Bornstein, 2003; Brewer, 2004; Force, 2008; ForsterLee, ForsterLee, Horowitz y King, 2006; Sommers y Ellsworth, 2009), la religión (Miller y Bornstein, 2006), el anonimato (e.g. Hazelwood y Brigham, 1998) y la influencia social (Marcus, Lyons y Guyton, 2000), la ideología política (e. g Najdowski, 2010; Shaw y Skolnick, 2005), y la personalidad (Shetowsky y Horowitz, 2004) de los miembros del jurado entre otras.

Encontramos además un gran número de estudios sobre la actuación del fiscal y la defensa (e.g Evans, Lee, y Lyon, 2009; Force, 2008; Higgins, Heath y Grannemann, 2007; Nelson, 2004; Shields, 2009), el testimonio del acusado y el arrepentimiento (Jehle, Miller y Kemmelmeir, 2009), características específicas del acusado (e.g. Antonio, 2006; Brewer 2004, Forsterlee et al., 2006; Mitchell, Haw, Pfeifer, Meissner, 2005) como el (Green, 1961; Mit-chell et.al, 2005; Nagel, 1969) status socioeconómico
(Force, 2008; Rose, Ellison y Diamond, 2008) y la reincidencia (Kalven y Zeisel, 1966, Hogarth, 1971), sin olvidar los estudios sobre la declaración de la víctima y características de la misma (Bottoms, Diviak, y Davis 1997; Schuller y Rzepa, 2002; Semmler y Brewer, 2002). En cuanto a las características tes al proceso de deliberación una de las áręs más estudiadas es la del efecto de los testigos expertos en el proceso de toma de decisión y el procesamiento de dicha información experta (e.g. Bright y GoodmanDelahunty, 2006; Finkelman, 2010; Krauss, Lieberman y Olson, 2004), los sesgos en la interpretación de la evidencia y testimonio experto (Carlson y Russo, 2001; Smith y Greene, 2005) y por último, el proceso de la toma de decisión del jurado (e.g. Antonio, 2006; Fitzgerald, 2000; ForsterLee et al., 2006; Louden y Skeem, 2007).

Si bien como indican Devine et al., (2001) las características de los participantes no poseen un valor probatorio en el estudio de la toma de decisión del jurado popular y por tanto no deberían influir en el veredicto, lo anteriormente expuesto nos sirve como marco de referencia para el presente estudio. Antes de proseguir creemos necesario establecer una diferenciación entre el jurado popular anglosajón (en este caso petit jury) y el Tribunal del Jurado español. A pesar de las aparentes similitudes entre ambos, los principios en los que se basan ambos son muy distintos. Mientras que en Estados Unidos es un derecho constitucional "ser juzgado por iguales" como se contempla en la sexta enmienda, en España es un derecho de los ciudadanos el participar en la justicia. Esto se traduce en una diferencia importante en cuanto a la selección de los miembros del jurado y por tanto el jurado en sí. Tal y como se expresa en la Constitución americana, el "tener derecho a un juicio justo y a ser juzgado por iguales" implica que el jurado anglosajón ha de ser representativo de la comunidad a la que pertenece el acusado y por tanto, la selección de los miembros del jurado ha de garantizar dicho derecho, mientras que en España, la selección de los miembros del jurado ha de garantizar el principio de igualdad para ejercer el derecho (y deber) de participar en la administración de justicia a través del Tribunal del Jurado. Por tanto, las características de los participantes, en 
especial los miembros del jurado, no adquieren tanta relevancia como las del procedimiento judicial. Debemos tener presentes varias cosas sobre el proceso de toma de decisión (deliberación y veredicto): Primero, a diferencia de los jueces y tribunales, el jurado está formado por un grupo no permanente de personas desconocidas, al que sus miembros pertenecen de manera involuntaria y anónima durante un espacio temporal limitado y no representan la postura de ningún colectivo (Soria, 2006). Segundo, una de las diferencias más importantes entre el jurado popular anglosajón y el español es que, mientras que el primero tiene como fin emitir un veredicto (y en ocasiones establecer la condena), el proceso de deliberación seguido y toma de decisión es secreto. Es decir, el jurado no debe dar una explicación sobre los elementos, pruebas, circunstancias y razonamiento utilizados para alcanzar dicho veredicto, mientras que en España, tal y como establece el Art. 61 de la Ley Orgánica 5/1995, el veredicto emitido por el jurado popular $\equiv$ e ser razonado, es decir, el jurado dehe explicar For qué ha alcanzado dicho veres. Esto, por tanto, hace que las variables personales de los participantes tengan menos peso en nuestro sistema judicial en cuanto a la toma de decisión se refiere. Por último, la última gran diferencia entre ambos jurados populares es que, a diferencia del jurado anglosajón, el jurado español es un órgano sometido a la ley y por tanto sus decisiones pueden ser recurridas, de aquí surge la necesidad de emitir un veredicto razonado y justificado.

En las últimas décadas ha crecido el interés por el estudio de los efectos de la participación en el jurado popular, los cambios de actitud tras la participación (e.g. Lamberth, Krieger, y Shay, 1982), la satisfacción de los miembros del jurado (Cutler y Hughes, 2001), actitudes y estereotipos (Louden y Skeem, 2007), conocimientos y actitudes (Consolini, 1992), percepciones sobre el sistema legal (Bornstein, Miller, Nemeth, Page y Musil, 2005), efectos de la participación en el tribunal del jurado a nivel de daños y estresores (Bernstein et al, 2005; Kaplan y Winget, 1992) y las creencias sobre la delincuencia (e. g Culhane, Hosch y Weaver, 2004; Haley, 2008; Naifach, 2002). Finalmente, respecto a la finalidad de la toma de decisión se han estableci- do tres tipos: rehabilitación, prevención o castigo. En la misma línea Diamond y Herbold (1981) manifiestan la existencia de dos tipos de decisiones, las retributivas cuyo objetivo principal es el castigo del delincuente por parte de la sociedad y las utilitarias dirigidas a la reducción del delito cuando la decisión judicial fuera capaz de promover la rehabilitación del delincuente como un instrumento de disuasión general o específica o, como una forma de incapacitación para delinquir. (e.g Najdowski, 2010)

En la actualidad, el Jurado español está instaurado como institución jurídica desde la Constitución Española de 1978, si bien este no llega a restablecerse hasta el año 1995 mediante la Ley Orgánica (LO) 5/1995, del 22 de mayo. No obstante, y partiendo de todo lo anterior, consideramos necesario el estudio sobre los cambios de conocimiento y opinión sobre el tribunal del jurado de los participantes del sistema judicial, al depender de ellos el veredicto final sobre el caso juzgado. Existe una notable falta de investigación sobre el impacto de participar en el jurado en los mismos participantes (三1, Black, Dees y Leichter, 2008).

El presente estudio se propone tres objetivos. Primero, comparar la opinión de los participantes sobre el jurado popular, antes y después de participar en un jurado en la sombra. Segundo, comparar los conocimientos de los participantes sobre el jurado popular antes y después de participar en el estudio para determinar si la experiencia influye en los mismos En tercer lugar, evaluar si los factores procesales influyen en la toma de decisión del jurado y la opinión de los participantes sobre el objetivo o finalidad de la misma.

\section{Metodología}

Un grupo de estudiantes universitarios seleccionados al azar entre los asistentes a la asignatura de Psicología Jurídica, tras informarles del carácter voluntario de su participación en el presente estudio, fueron citados en el Palacio de Justicia de la Audiencia Provincial de Barcelona para participar en una experiencia de jurados. A partir de dicho momento se siguió el procedimiento establecido en la legislación vigente. 


\section{Muestra}

La muestra del estudio fue seleccionada entre estudiantes del último curso de la licenciatura de Psicología. Se seleccionaron al azar, entre aquellos que cumplieran la condición de no tener conocimientos específicos sobre el funcionamiento o condicionantes del jurado. Tampoco debían poseer experiencia personal como miembros de jurados reales. Todos los seleccionados aceptaron la participación voluntaria en la experiencia. Previamente al inicio de la experiencia desconocían el tipo de caso a juzgar y la naturaleza concreta del estudio.

La muestra final del estudio estaba compuesta por 19 Ss, 14 mujeres $(73.7 \%)$ y 5 hombres $(26.3 \%)$ de edades comprendidas entre los 21 y 48 años, hallándose el $57.9 \%$ entre los 21 y los 24 años y de nivel sociocultural medio-alto. Respecto a la preferencia política, $4 \mathrm{Ss}$ no contestaron la pregunta. $8 \mathrm{Ss}$ $(53.3 \%)$ se declararon nacionalistas y el resto próximos a partidos estatales. Por otra parte, 10Ss $(66.6 \%)$ se situaban próximos a una ideología de izquierdas, frente al resto de derechas.

Ocho participantes de la muestra inicial $(n=27)$ no finalizaron el estudio debido a cuestiones de incompatibilidad horaria al prolongarse el juicio durante más de cuatro días y en horario de mañana y tarde.

\section{Instrumentos}

Los participantes contestaron a un cuestionario elaborado ah hoc: "Cuestionario de los Efectos de la Participación en un Jurado (CEPJ)" antes y después de participar en el estudio, dividido en dos bloques: uno relativo a la opinión y actitud hacia el Tribunal del Jurado (creación, implicaciones de su implementación, toma de decisiones, causa del delito, prioridad del veredicto) y otro sobre los conocimiento acerca del Tribunal del Jurado (tipos de delitos enjuiciados, composición, proceso de toma de decisión. y efectos de la decisión).

\section{Procedimiento}

Tras ser citados a las ocho de la mañana en la Audiencia Provincial de Barcelona, se les informó de la naturaleza del estudio (formar parte de un jurado a la sombra de forma paralela al tribunal del jurado oficialmente constituido), del carácter voluntario de su participación y la posibilidad de abandonar la experiencia en cualquier momento. Tras quedar constituido el "jurado a la sombra" se procedió a administrar el "Cuestionario de los Efectos de la Participación en un Jurado (CEPJ)".

A las nueve de la mañana los jurados constituidos fueron reunidos en una Sala del Palacio de Justicia donde el Presidente-Magistrado de la Sala y por tanto, Presidente del Tribunal del Jurado, les informó sobre el procedimiento general a seguir y de sus funciones según la Ley del Tribunal del Jurado. Al igual que el jurado real, el jurado a la sombra asistió a la vista oral, presenciando la lectura de los escritos de calificación, la declaración del imputado, testigos, policías y peritos, presentación de documentos, etc. El juicio se prolongó durante seis horas (mañana y tarde). Al finalizar este, el Magistrado Presidente se reunió con los participantes y les explicó los criterios-marco del veredicto, las cuestiones sobre las que debían pronunciarse como jurado, las reglas que rigen la deliberación y votación y la forma en la que debían emitir su veredicto. Acto seguido, los participantes fueron reunidos en una sala aparte y divididos en dos grupos para llevar a cabo la deliberación y alcanzar un veredicto. Solicitaron la presencia del Magistrado Presidente en dos ocasiones para realizar aclaraciones sobre el caso. Una vez alcanzado el veredicto, los participantes contestaron una vez más el "Cuestionario de los Efectos de la Participación en un Jurado (CEPJ)". Tras agradecerles su participación se dio por finalizada la experiencia.

\section{Caso enjuiciado ${ }^{1}$}

\section{Características del Caso}

El caso enjuiciado fue el asesinato de una mujer a manos de su marido. La pareja llevaba casada aproximadamente dos años y tenía un hijo. En el momento de los hechos estaban separados desde hacía un mes. No existían antecedentes de violencia doméstica previa, pero si episodios de acoso previos a la muerte. Tanto víctima como acusado eran de clase social baja.

\footnotetext{
${ }^{1}$ Algunos datos han sido modificados para evitar la posible identificación de los participantes.
} 
Los hechos sucedieron cuando la mujer regresaba a su domicilio y el acusado la seguía durante el último trayecto por la calle hablando con ella.

\section{Características del acusado}

Hombre joven con un grado importante de invalidez debida a un accidente previo. Apuñala a su mujer 31 veces y luego intenta suicidarse. No tiene antecedentes penales previos.

\section{Características de la víctima}

Mujer joven de gran atractivo físico, unos años mayor que su marido. Se dedica a la prostitución antes de conocerlo y tiene antecedentes penales previos.

\section{Análisis de datos}

Los resultados fueron analizados con SPSS 15 para Windows ${ }^{\circledR}$. La opinión y los conocimientos de los participantes sobre el Tribunal de Jurado fueron analizadas a través de comparaciones de medias y proporciones con medidas repetidas.

\section{Resultados}

\section{Opinión sobre el Tribunal del Jurado}

Respecto a la instauración del jurado popular, aunque no hallamos una diferencia estadística significativa, los resultados mostraron un incremento porcentual de su aceptación (78.9\% - 89.5\%) tras la experiencia, mientras que la opinión sobre la participación en un jurado lar no mostró variaciones significativas, observánçse un leve descenso tras la experiencia $(89.5 \%-84.2 \%)$.

\section{Implicaciones de la implantación del Jurado}

Para llevar a cabo el análisis, subdividimos esta variable en seis subgrupos: incremento de la participación popular en la justicia, mayor efectividad de la decisión del Tribunal del Jurado que la de los jueces, incremento de los gastos judiciales, cumplimiento de la normativa de la Constitución, incremento de los retrasos en la justicia y la provocación de un enfrentamiento con la organización judicial. No hallamos cambios significativos en ninguna de las variables tras la experiencia.

\section{Toma de decisión}

Dividimos este apartado en tres variables: factores procesales, características personales del acusado y objetivos de las decisiones tomadas. A su vez, dividimos la variable factores procesales en cinco indicadores (actuación del fiscal, de la defensa, declaración del acusado, de los testigos y peritos). Sólo el indicador "actuación de la defensa" mostró cambios significativos $(\mathrm{p}=0.043)$, considerándose menos relevante tras la experiencia. La Tabla 1 muestra los resultados del análisis de los factores procesales.

\section{Características personales del acusado}

Subdividimos las características en género, raza,

Tabla 1. Análisis de los factores procesales

\begin{tabular}{lcccccc}
\hline FACTORES PROCESALES & $\boldsymbol{X}_{\boldsymbol{1}}$ & $\boldsymbol{S D}_{\boldsymbol{1}}$ & $\boldsymbol{X}_{\mathbf{2}}$ & $\boldsymbol{S \boldsymbol { D } _ { 2 }}$ & $\boldsymbol{p}$ & Nivel de Significación \\
\hline Fiscal & 3.32 & 1.29 & 2.74 & 1.24 & .086 & N.S $(*)$ \\
Defensa & 3.37 & 1.21 & 2.58 & 1.46 & .043 & .288 \\
Inculpado & 3.26 & 1.37 & 2.84 & 1.54 & .05 & N.S. \\
Testimonios & 2.74 & 1.10 & 2.68 & 1.53 & .875 & N.S. \\
Peritos & 3.26 & 1.56 & 2.63 & 1.42 & .117 & N.S. \\
\hline
\end{tabular}

(*) N.S.=No Significativo 
estatus social, reincidencia, trastornos mentales, consumo de drogas y arrepentimiento espontáneo. Los resultados no mostraron diferencias significativas tras la experiencia. No obstante, el trastorno mental y el consumo de drogas puntuaron $\Longrightarrow$ las más relevantes antes y después de participár en el estudio.

\section{Objetivos de la decisión jurídica}

Subdividimos los objetivos en: rehabilitación, prevención y castigo. La rehabilitación del agresor fue la única que se mostró estadísticamente significativa tras la experiencia $(\mathrm{p}=.004)$ pasando a ser la menos valorada tras la experiencia como objetivo de la toma de decisión jurídica (Tabla.2).

\section{Origen del delito}

El análisis de los factores relativos al origen del delito (delincuente, drogas, supervivencia, sociedad, distribución equitativa de riquezas, no-voluntad de trabajar, problemas familiares, intrínseco del delin- cuente y aprendizaje) revelaron que los participantes consideraron la "supervivencia" la causa principal de la delincuencia antes de la experiencia. Esta se definía como la creencia por la cual las personas necesitan sobrevivir, y algunas veces, el delito es la única alternativa. Tras participar en el jurado lar la supervivencia fue menos valorada $(\mathrm{p}=$ \&, 3 ) mientras que los problemas familiares fueron considerados la causa principal atribuida al origen del delito (Tabla.3).

\section{Interés prioritario del veredicto}

Respecto al interés prioritario del veredicto (víctima, acusado y sociedad), ninguna mostró cambios significativos. Tanto antes como después de la experiencia, los participantes consideraron que la finalidad del veredicto debía estar orientada hacia la víctima.

En el segundo grupo de análisis evaluamos el cambio de conocimiento de los participantes respecto al jurado popular donde estudiamos cuatro variables:

Tabla 2. Análisis de los objetivos de las decisiones judiciales

\begin{tabular}{|c|c|c|c|c|c|c|c|}
\hline Objetivos & & $X_{1}$ & $S D_{1}$ & $X_{2}$ & $S D_{2}$ & $p$ & Significación \\
\hline \multirow[t]{2}{*}{ Rehabilitación } & Rehabilitar al agresor & 3.6 & 1.9 & 2.6 & 1.7 & 0.004 & $<0,005$ \\
\hline & Resocializar al penado & 3.4 & 1.6 & 3.4 & 1.8 & 0.9 & N.S $(*)$ \\
\hline \multirow[t]{4}{*}{ Prevención } & Ejemplarizar a futuros delincuentes & 4.1 & 1.8 & 3.3 & 1.8 & 0.1 & N.S. \\
\hline & Evitar reincidencia del acusado & 3.6 & 1.9 & 3.9 & 2.2 & 0.5 & N.S. \\
\hline & Prevenir el delito & 3.8 & 2.0 & 3.1 & 2.1 & 0.1 & N.S. \\
\hline & Evitar el delito temporalmente & 4.1 & 2.0 & 4.3 & 2.0 & 0.6 & N.S. \\
\hline \multirow[t]{2}{*}{ Castigo } & Castigar al culpable & 4.3 & 1.9 & 4.3 & 2.0 & 1.0 & N.S. \\
\hline & Castigar al penado & 4.7 & 2.0 & 4.1 & 2.1 & 0.3 & N.S. \\
\hline
\end{tabular}

(*) N.S. = No Significativo

Tabla 3. Análisis del origen del delito

\begin{tabular}{|c|c|c|c|c|c|c|}
\hline ORIGEN DEL DELITO & $X_{1}$ & $S D_{1}$ & $X_{2}$ & $S D_{2}$ & $p$ & Nivel de Significación \\
\hline Delincuentes & 4.8 & 1.4 & 4.7 & 2.2 & 0.8 & N.S $(*)$ \\
\hline Drogas & 5.0 & 1.5 & 4.7 & 1.9 & 0.6 & N.S. \\
\hline Supervivencia & 3.6 & 1.8 & 2.5 & 1.8 & 0.03 & $<0.05$ \\
\hline Sociedad & 4.9 & 1.5 & 4.3 & 2.1 & 0.3 & N.S. \\
\hline Distribución equitativa de riquezas & 2.3 & 1.1 & 2.3 & 1.4 & 1.0 & N.S. \\
\hline No voluntad de trabajar & 3.3 & 1.8 & 2.7 & 1.8 & 0.2 & N.S. \\
\hline Problemas familiares & 5.7 & 0.9 & 4.9 & 1.5 & 0.2 & N.S. \\
\hline Intrínseco al delincuente & 2.4 & 1.2 & 2.2 & 1.1 & 0.4 & N.S. \\
\hline Aprendizaje & 4.1 & 1.6 & 4.1 & 1.7 & 1 & N.S. \\
\hline
\end{tabular}

(*) N.S. =No Significativo 
En la primera, tipo de delitos competencia del Tribunal del Jurado, observamos un desconocimiento de las causas susceptibles a ser enjuiciadas por el mismo. Antes de la experiencia, el delito de lesiones fue considerado competencia propia por el $89.5 \%$ de los participantes $(\mathrm{n}=17)$ seguido por el económico, ecológico, violación y abuso sexual (73.7\%). 13 participantes (68.4\%) indicaron el homicidio como competencia del jurado popular

Tabla 4. Tipos de delitos competentes del Tribunal Jurado

\begin{tabular}{lrrrr}
\hline ALTERNATIVAS & $\mathbf{n}_{\mathbf{1}}$ & $\mathbf{\%}$ & $\mathbf{n}_{\mathbf{2}}$ & $\boldsymbol{\%}$ \\
\hline Terrorismo & 5 & 26.3 & 8 & 42.1 \\
Económicos & 14 & 73.7 & 11 & 57.9 \\
Ecológicos & 14 & 73.7 & 14 & 73.7 \\
Homicidio & 13 & 68.4 & 15 & 78.9 \\
Lesiones & 17 & 89.5 & 15 & 78.9 \\
Violación & 14 & 73.7 & 15 & 78.9 \\
Abuso Sexual & 14 & 73.7 & 14 & 73.7 \\
Contra la propiedad & 11 & 57.9 & 11 & 57.9 \\
Todos los delitos & 7 & 36.8 & 8 & 42.1 \\
\hline
\end{tabular}

No se observaron cambios significativos en los resultados tras la experiencia. Cabe destacar que sólo un $10.5 \%$ de los participantes $(\mathrm{n}=2)$ modificó su respuesta en cuanto al homicidio incluyéndolo en las competencias del tribunal del jurado, siendo este el caso enjuiciado. Del mismo modo, debemos destacar el aumento en el número de participantes que consideraron los casos de terrorismo como competencia del Tribunal del Jurado, y la ausencia de cambio en casos como la violación, abuso sexual o "todos los delitos penados" tras haber participado en el estudio.

Respecto al número de componentes del Tribunal Jurado inicialmente el $84.2 \%$ de los participantes fue capaz de citar su composición en 9 miembros. Tras la experiencia todos los participantes dieron la cifra correcta. A pesar de no observarse diferencias estadísticas significativas destaca el cambio del conocimiento, pasando de una mayoría a la totalidad de la muestra.

El procedimiento para tomar la decisión jurídica por el Jurado se dividió en tres categorías (unanimidad, mayoría absoluta y mayoría relativa). A pesar de no haber cambios estadísticamente significativos debemos destacar el cambio de opinión sobre la mayoría absoluta.
Tabla 5. Proceso de toma de decisiones

\begin{tabular}{lrcrc}
\hline ALTERNATIVAS & $\mathbf{n}_{\mathbf{1}}$ & $\boldsymbol{\%}$ & $\mathbf{n}_{\mathbf{2}}$ & $\boldsymbol{\%}$ \\
\hline Unanimidad & 3 & 15.8 & 3 & 15.8 \\
Por Mayoría Absoluta & 14 & 73.7 & 12 & 63.2 \\
Por Mayoría Relativa & 2 & 10.5 & 2 & 10.5 \\
NS/NC & 0 & 0 & 2 & 10.5 \\
\hline TOTALES & 19 & 100 & 19 & 100
\end{tabular}

Los efectos de la decisión del Tribunal del Jurado sobre la pena del inculpado la declaración de culpabilidad, las eximentes/agravantes de responsabilidad o combinación pueden consultarse en la Tabla 6. El análisis reveló una diferencia significativa en las tres variables tras la experiencia $(\mathrm{p}=0.004)$ mostrando un conocimiento más ajustado sobre el proceso de toma de decisión del jurado. Inicialmente los participantes consideraron la "declaración de culpabilidad" como la tarea principal del jurado, pasando a considerarse posteriormente la "declaración de culpabilidad" y las "eximentes/agravantes".

Tabla 6. Decisiones del Tribunal del Jurado

\begin{tabular}{|c|c|c|c|c|}
\hline ALTERNATIVAS & $n_{1}$ & $\%$ & $\mathbf{n}_{2}$ & $\%$ \\
\hline $\mathrm{De} \equiv$ Jión & 10 & 52.6 & 1 & 5.3 \\
\hline EximR fites/agravantes & 1 & 5.3 & 1 & 5.3 \\
\hline Pena x Declaración & 0 & 0 & 3 & 15.8 \\
\hline Pena x Eximentes/Agravantes & 0 & 0 & 1 & 5.3 \\
\hline $\begin{array}{l}\text { Declaración x Eximentes/ } \\
\text { agravantes }\end{array}$ & 6 & 31.6 & 10 & 52.6 \\
\hline Pena x Declaración $x$ & & & & \\
\hline Eximentes/Agravantes & 2 & 10.5 & 3 & 15.8 \\
\hline TOTAL & 19 & 100 & 19 & 100 \\
\hline
\end{tabular}

\section{Conclusiones}

Al principio de la experiencia los participantes calificaron como legítima la creación del Tribunal del Jurado mostrando un ligero incremento de dicha opinión tras la finalización de la misma. A pesar de ello, la mayoría indicó no desear formar parte del jurado popular en un futuro debido a factores causales contextuales (problemas en el ámbito laboral) o sociales (aislamiento y cantidad de tiempo invertido). Estos resultados respaldan estudios anteriores donde la opinión de los participantes sobre el sistema judicial no cambió al participar en un jurado popular (e.g. Cutler y Hughes, 2001) viéndose refle- 
jado en la ausencia de cambios de opinión sobre los efectos de la implantación del jurado, origen del delito e interés prioritario del veredicto.

Si bien observamos cambios en los factores procesales (i.e actuación de la defensa) y una ausencia de cambio e influencia en cuanto a las características del acusado en la toma de decisión, esto debe interpretarse en el marco de nuestro sistema legal y características del Tribunal del Jurado. No sólo la participación en la experiencia judicial no tuvo influencia sobre las variables personales del acusado sino que además, dicha influencia disminuyó respecto a la condición inicial. Así los factores "trastorno mental" y "arrepentimiento espontáneo" fueron valorados positivamente. Todo ello es atribuible a las especificidades del caso juzgado. En dicho sentido destaca el factor consumo de drogas donde observamos una ligera disminución de su importancia tras la experiencia (Haque y Cumming, 2003), al igual que la disparidad en las opiniones sobre el origen del delito donde se contemplan causas muy diversas desde problemas familiares a procesos de aprendizaje social.

Respecto a los objetivos de la toma de decisión jurídica observamos como se le atribuye esencialmente una función de castigo, hecho que contradice la meta última de nuestro sistema judicial basado en la rehabilitación y no el castigo. Esto y el cambio de opinión significativo sobre la función de rehabilitar al agresor pudiendo estar estrechamente relacionado con el caso enjuiciado.

En el segundo grupo de resultados relativos al grado de conocimiento sobre el Jurado observamos una mejoría en lo relativo al número de sus componentes y la decisión jurídica (declaración de culpabilidad/ inocencia y eximentes/ agravantes). Contrariamente, en otros aspectos como la regla decisoria no se produjeron los resultados esperados.

Respecto al conocimiento del Jurado, si bien pudimos confirmar la existencia de un proceso de aprendizaje, este no fue homogéneo, ni afectó al conjunto del funcionamiento del órgano del Jurado. En lo relativo a la regla decisoria podría atribuirse a una falta de atención de los participantes, a una explicación o comprensión deficiente por parte de los "instructores" o una confusión conceptual fruto de factores lingüísticos (Chilton y $\mathrm{H} \equiv$

\section{Limitaciones}

Si bien los estudios de campo con jurados reales, en nuestro caso el jurado a la sombra, son más realistas y sus resultados son más generalizables, conllevan la limitación de tener que trabajar con muestras pequeñas.

\section{Futuras Investigaciones}

Consideramos necesario llevar a cabo el estudio con diferentes casos judiciales lo que nos permitiría establecer si la presencia o ausencia de cambios en la opinión de los participantes se debe al tipo de caso enjuiciado, a las formas de interacción propias de un grupo con características especiales como las del jurado o simplemente a las características individuales de los participantes. Por otra parte, sería interesante evaluar el grado de satisfacción con el sistema judicial de los participantes.

\section{Referencias}

Abshire, J., y Bornstein, B. (2003). "Juror sensitivity to the cross-race effect. Law and Human Behavior, 27, 471-480.

Antonio, M. E. (2006). Arbitrariness and the death penalty: how the defendant's appearance during trial influences capital jurors' punishment decision. Behavioral Sciences y The Law, 24, 215-234.

Bornstein, B., Miller, M., Nemeth, R., Page, G., y Musil, S. (2005). Juror reactions to jury duty: perceptions of the system and potential stressors. Behavioral Sciences and The Law, 23, 321-346.

Bottoms, B. L., Diviak, K., y Davis, S. (1997). Jurors' reactions to satanic ritual abuse allegations. Child Abuse y Neglect, 21, 845-859.

Brewer, T. W. (2004). Race and jurors' receptivity to mitigation in capital cases: the effect of jurors', defendants', and victims' race in combination. Law and Human Behavior, 28, 529-545.

Bright, D., y Goodman-Delahunty, J. (2006). Gruesome Evidence and Emotion: Anger, Blame, and Jury Decision-Making. Law and Human Behavior, 30 , 183-202. 
Carlson, K., y Russo, J. (2001). Biased interpretation of evidence by mock jurors. Journal of Experimental Psychology: Applied, 7, 91-103.

Collett, M. E., \& Kovera, M. B. (2003). The effects of British and American trial procedures on the quality of juror decision making. Law and Human Behavior, 27, 403-422.

Consolini, P. (1992). Learning by doing justice: Private jury service and political attitudes. Doctoral dissertation, University of California at Berkeley.

Culhane, S., Hosch, H., y Weaver, W. (2004). Crime Victims Serving as Jurors: Is There Bias Present?.Law and Human Behavior, 28 , 649-659.

Cutler, B. L., y Hughes, D. M. (2001), Judging jury service: Results of the north Carolina administrative office of the courts juror survey. Behavioral Sciences $y$ the Law, 19, 305-320. doi: 10.1002/ bsl.439.

Daftary-Kapur, T., Dumas, R., y Penrod, S. (2010). Jury decision-making biases and methods to counter them. Legal and Criminological Psychology, 15, 133-154.

Devine, D., Clayton, L., Dunford, B., Seying, R., y Price (2001). Jury Decision Making, : 45 Years of Empirical Research on Deliberating Groups. Psychology, Public Policy, and Law, 7, 622-727.

Diamond, S., y Herbold C. (1981). Supplemental report-adult pre-realease facilities. Volume $V$ of American Prisons ans Jails. Washington, D.C: U.S. Department of Justice.

España. Ley Orgánica 10/1995, de 23 de Noviembre, del Código Penal. Boletín Oficial del Estado, 24 de noviembre de 1995, núm.281, 33987-34058. Disponible en: http://www.boe.es/ aeboe/consultas/bases_datos/doc.php?id=BOEA-1995-25444.

España. Ley Orgánica 5/1995, de 22 de Mayo, del Tribunal del Jurado. Boletín Oficial del Estado, 5 de agosto de 1995, núm. 186, 24254-24256. Disponible en: http://www.boe.es/aeboe/consultas/bases_datos/doc.php?id=BOE-A-199518778.

Evans, A., Lee, K., y Lyon, T. (2009). Complex questions asked by defense lawyers but not prosecutors predicts convictions in child abuse trials. Law and Human Behavior, 33, 258-264.
Finkelman, J. (2010). Litigation consulting: Expanding beyond jury selection to trial strategy and tactics. Consulting Psychology Journal: Practice and Research, 62, 12-20. doi:10. 1037/a0018648.

Fitzgerald, J. M. (2000). Younger and Older Jurors. The Journals of Gerontology Series B: Psychological Sciences and Social Sciences, 55, 323-331.

Force, N. (2008) Factors Influencing Juror Sentencing Decisions: Race, Social Economic Status, Attorney Credibility and the Relevance of Stereotype Attribution Theory. Boca Raton: Dissertations.com.

Forsterlee, L., Horowitz, I. A. y Bourgeois, M. J. (1993). Juror Competence in Civil Trials: Effects of Preinstruction and Evidence Technicality. Journal of Applied Psychology, 78, 14-21.

Forsterlee, R., Forsterlee, L., Horowitz, I., y King, E. (2006). The effects of defendant race, victim race, and juror gender on evidence processing in a murder trial. Behavioral Sciences and The Law, 24, 179-198.

Golding, J., Bradshaw, G., Dunlap, E., y Hodell, E. (2007). The impact of mock jury gender composition on deliberations and conviction rates in a child sexual assault trial. Child Maltreatment, 12, 182-190.

Green, E. (1961). Judicial attitudes in sentencing: a study of the factors underlying the sentencing practices of the criminal court of Philadelphia. London: Macmillan.

Haley, M. (2008). Just world beliefs, ethnicity, and juror bias. Dissertation Abstracts International, 69.

Haque, Q., y Cumming, I. (2003) Intoxication and legal defenses. Advances in Psychiatric Treatment, 9, 144-151.

Hazelwood, D. L., y Brigham, J. C. (1998). The Effects of Juror Anonymity on Jury Verdicts. Law and Human Behavior, 22, 695-713.

Higgins, P. L., Heath, W.P., y Grannemann, B. D. (2007). How type of excuse defense, mock juror age, and defendant age affect mock jurors' decisions. Journal of Social Psycholy, 147, 371-392.

Hogarth, J. (1971). Sentencing as a human process. Toronto: University of Toronto Press.

Jehle, A., Miller, M., y Kemmelmeier, M. (2009). The Influence of Accounts and Remorse on Mock 
Jurors' Judgments of Offenders. Law and Human Behavior, 33, 393-404.

Kalven, H., y Zeisel, H. (1966). The American jury. Chicago: University of Chicago press.

Kaplan, S. M., y Winget, C. (1992). The occupational hazards of jury duty. Bulletin of the American Academy of Psychiatry Law, 20, 325-333.

Krauss, D., Lieberman, J., y Olson, J. (2004). The effects of rational and experiential information processing of expert testimony in death penalty cases. Behavioral Sciences and The Law, 22, 801822.

Lamberth, J., Krieger, E., y Shay, S. (1982). Juror decision making: A case of attitude change mediated by authoritarianism. Journal of Research in Personality, 16, 419-434.

Louden, J. E., y Skeem, J. L. (2007). Constructing insanity: jurors' prototypes, attitudes, and legal decision-making. Behavioral Sciences and the Law, 25, 449-470.

Marcus, D., Lyons, P., y Guyton, M. (2000). Studying Perceptions of Juror Influence In Vivo: A Social Relations Analysis. Law and Human Behavior, 24, 173-186.

Miller, M. K., y Bornstein, B. H. (2006). The use of religion in death penalty sentencing trials. Law and Human Behavior, 30, 675-684.

Mitchell, T., Haw, R., Pfeifer, J., y Meissner, C. (2005). Racial Bias in Mock Juror DecisionMaking: A Meta-Analytic Review of Defendant Treatment. Law and Human Behavior, 29, 621-637.

Nagel, S. (1969). The legal process from a behavioral perspective. Homewood: Doney Press.

Naifach, H. (2002, March). Jury deliberation style and just world belief. Dissertation Abstracts International Section A, 62.

Najdowski, C. (2010). Jurors and social loafing: Factors that reduce participation during jury deliberations.American Journal of Forensic Psychology, 28, 39-64.

Nelson, M. (2004). The effect of attorney gender on jury perception and decision-making. Law y Psychology Review, 28, 177-193.
Newcombre, P. A., y Bransgrove, J. (2007). Perceptions of witness credibility: variations across age. Journal of Applied Developmental Psychology, $28,318-331$.

Rose, M., Ellison, C., y Diamond, S. (2008). Preferences for juries over judges across racial and ethnic groups. Social Science Quarterly, 89, 372-391.

Schuller, R., y Rzepa, S. (2002). "Expert testimony pertaining to battered woman syndrome: its impact on jurors' decisions." Law and Human Behavior, 26, 655-673.

Semmler, C., y Brewer, N. (2002). Effects of mood and emotion on juror processing and judgments. Behavioral Sciences and the Law, 20, 423436.

Shaw, J., y Skolnick, P. (2005). Effects of psycholegal knowledge on decision-making by mock juries.Applied Psychology in Criminal Justice, 1, 90-109.

Shetowsky, D., y Horowitz, L. (2004) How the Need for Cognition Scale Predicts Behavior in Mock Jury Deliberations Law and Human Behavior, 28, 305-337.

Shields, C. (2009). An analysis of prosecutorial and defense strategies in federal terrorism trials from 1980 to 2004. Dissertation Abstracts International Section A, 69.

Sommers, S. R. (2006). On racial diversity and group decision making: identifying multiple effects of racial composition on jury deliberations. Journal of Personality Social Psychology, 90, 597-612.

Smith, A. C., \& Greene, E. (2005). Conduct and its consequences: Attempts at debasing jury judgments. Law and Human Behavior, 29, 505-526.

Sommers, S., y Ellsworth, P. (2009). 'Race salience' in juror decision-making: Misconceptions, clarifications, and unanswered questions. Behavioral Sciences y the Law, 27, 599-609.

Soria Verde, M.A. (2006). Manual de Psicología Jurídica e Investigación Criminal. Madrid: Pirámide.
Manuscrito recibido:

Revisión recibida:

Aceptado: 